\title{
Reaction condition optimization for non-oxidative conversion of methane using artificial intelligence
}

Hyun Woo Kim,,${ }^{1 \dagger *}$ Sung Woo Lee,,$^{2 \dagger}$ Gyoung S. Na, ${ }^{1 \dagger}$ Seung Ju Han, ${ }^{2}$ Seok Ki Kim,, 3 Jung Ho Shin, ${ }^{1}$ Hyunju Chang, ${ }^{1 *}$ Yong Tae Kim, ${ }^{2 *}$

${ }^{1}$ Chemical Data-Driven Research Center, Korea Research Institute of Chemical Technology (KRICT), Daejeon 34114, Korea

${ }^{2} \mathrm{C} 1 \mathrm{Gas} \&$ Carbon Convergent Research Center, Korea Research Institute of Chemical Technology, Daejeon 34114, Republic of Korea

${ }^{3}$ Advanced Materials and Chemical Engineering, University of Science \& Technology, Daejeon 34113, Republic of Korea

${ }^{\dagger}$ These authors contributed equally to this work.

*E-mail address: ahwk@krict.re.kr, hjchang@krict.re.kr, ytkim@krict.re.kr 


\begin{abstract}
Chemical reactions typically have numerous controllable factors that need to be optimized to yield the desired products. Although traditional experimental methods are limited to explore possible combinations of these factors, artificial intelligence (AI) can provide the optimal solution based on chemical reaction data. In this study, we optimize the non-oxidative conversion of methane to $\mathrm{C}_{2}$ compounds using $\mathrm{AI}$, such as machine learning (ML) to predict experimental results and metaheuristics to optimize reaction conditions. A decision tree-based machine learning method can reasonably predict the reaction outcomes $\left(\mathrm{CH}_{4}\right.$ conversion, $\mathrm{C}_{2}$ yield, and selectivities for $\mathrm{C}_{2}$ and coke) with an error of $<5 \%$. Trained ML models are applied to maximize the $\mathrm{C}_{2}$ yield by optimizing the reaction parameters with metaheuristics. We can simultaneously enhance the $\mathrm{C}_{2}$ yield and suppress the coke formation by improving the multi-objective function for the optimization. We believe that our method will be helpful to optimize the chemical reaction conditions with multiple targets.
\end{abstract}

\title{
Table of Contents
}

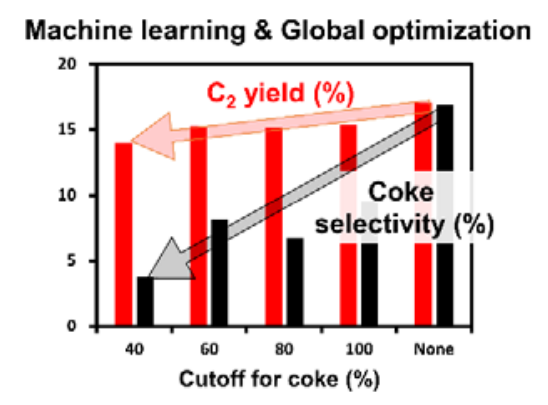

Using machine learning and global optimization, we optimize reaction conditions for non-oxidative conversion of methane. 


\section{Introduction}

Numerous factors govern chemical reactions and some of the factors can be artificially manipulated to optimize the yield of desired products. In the presence of catalysts, engineering these factors for chemical reactions is required to improve the catalysts as well as reaction conditions for the best performance. ${ }^{1}$ Due to the complexity of catalytic processes, numerous approaches have been employed, such as high-throughput methods, ${ }^{2,3}$ data-mining, ${ }^{4-6}$ and genetic algorithms. $^{7-9}$ Although these approaches have been successfully applied to design new heterogeneous catalysts, machine learning (ML) methods have recently attracted considerable attention as they require smaller datasets and lower computational costs than traditional methods.

${ }^{10}$ Moreover, in chemical reaction engineering, ML methods can play an essential role, for example, in self-optimizing platforms ${ }^{11}$ and suggesting reaction conditions. ${ }^{12}$

ML can match input variables (or features) to target properties and thus can be generally employed to available datasets for numerous catalytic reactions. ${ }^{4,13-15}$ For example, successful applications of ML methods toward the optimization of heterogeneous catalysts are shown for a database of oxidative coupling of methane (OCM) which contains $\sim 1800$ catalysts and reaction conditions. ${ }^{4}$ With the advancements in data science, chemical reaction data can be investigated with several approaches such as statistical analysis, ${ }^{4}$ machine learning. ${ }^{10,}{ }^{16}, 17$ micro-kinetic simulations, ${ }^{18}$ and meta-analysis. ${ }^{19}$ Moreover, a recent experimental study demonstrated that the combination of high-throughput experiments and ML techniques can provide catalyst compositions with improved catalytic efficiencies for the OCM with less effort. ${ }^{20,21}$

In chemistry-related fields, the developments of OCM catalysts is important as methane is abundant in natural gas ${ }^{22}$ and is easily found in biomass. ${ }^{23,24}$ Considering its importance, several processes have been proposed for the activation of methane molecules, such as steam reforming, ${ }^{25}$ 
dry reforming, ${ }^{26}$ and oxidative ${ }^{27}$ and non-oxidative ${ }^{28}$ coupling of methane. Recently, non-oxidative coupling of methane (NOCM) or methane to olefins, aromatics, and hydrogen (MTOAH) has attracted considerable attention ${ }^{29,30}$ because it does not require any oxidizing reagents, which potentially transform methane to oxidized products such as $\mathrm{CO}_{2}$. Although undesirable oxidation reactions do not occur in MTOAH unlike OCM, the application of MTOAH is still limited as it occurs through thermodynamically unfavorable processes toward hydrocarbons. ${ }^{31}$ Several strategies with external energy sources such as heat. ${ }^{28,32-34}$ photon, ${ }^{35,36}$ and plasma ${ }^{37,38}$ have been proposed to overcome this limitation.

The main challenge of MTOAH is the maximum conversion of methane possible while achieving high energy efficiency. The initial $\mathrm{C}-\mathrm{H}$ bond activation of methane requires the highest energy $\left(425 \mathrm{~kJ} \mathrm{~mol}^{-1}\right)$ among other steps. Thus, controlling the reaction pathways toward target products is quite challenging. ${ }^{39}$ Thermodynamics favors coke formation under radical-mediated conditions, requiring a precise design of lateral active sites, ${ }^{40}$ catalytic systems,${ }^{41}$ and reaction engineering. ${ }^{42,43}$ Experimentally, an MTOAH system facilitates numerous improvements in terms of conversion and selectivity (i.e., methane conversion of $48 \%$ and ethylene selectivity of $50 \%$ using a silica-lattice-confined single Fe atom) ${ }^{30}$ However, the lack of theoretical understanding may hinder the development of breakthrough technologies in this field. According to our previous study, although the MTOAH reaction is dominated in the gas phase, a proper use of the catalytic surface can improve the reaction activity toward the efficient formation of the hydrocarbon products. $^{29}$

To maximize the yield of the desired product in the MTOAH reaction proceeding at a high temperature, it is necessary to simultaneously consider the catalytic reaction ${ }^{44}$ and gas-phase radical reaction. However, despite the significant contribution of the gas-phase reaction to the 
product distribution, no chemical method, other than changing operation conditions, has been reported to control its kinetics. Moreover, as the gas-phase reaction is a set of numerous radical chain reactions, it is almost impossible to determine the optimal reaction conditions by simulating each individual reaction. Therefore, it is essential to accumulate information on gas-phase methane pyrolysis and optimize operating conditions for further improvement of the catalytic system.

To this end, we employed a hybrid approach by combining supervised ML models and metaheuristics-based optimizations ${ }^{45}$ to enhance the MTOAH to $\mathrm{C}_{2}$ molecules. Adopting an idea that artificial intelligence (AI) is a broad concept, which includes machine learning, ${ }^{46}$ we will shortly denote our hybrid approach as an AI method. Experimentally, we chose a quartz tubular reactor with a crystalline $\mathrm{SiO}_{2}$ surface, which can balance gas-phase reactions. We then constructed ML models to predict experimental results. A decision tree-based method ${ }^{47}$ could reasonably predict the reaction outcomes $\left(\mathrm{CH}_{4}\right.$ conversion, $\mathrm{C}_{2}$ yield, and selectivities for $\mathrm{C}_{2}$ and coke) with an error of $<5 \%$. After establishing ML models, we maximized the $\mathrm{C}_{2}$ yield by optimizing the experimental input variables using metaheuristics. ${ }^{45}$ We also experimentally demonstrated that the $\mathrm{C}_{2}$ yield can be enhanced without increasing the coke formation with $\mathrm{AI}$ acting as a guide. We believe that our method can be useful for optimizion of chemical reaction conditions with multiple targets.

\section{Methods}

\section{Experiment}

MTOAH was carried out in a quartz tubular reactor $($ length $=150 \mathrm{~cm}$ ) with various inner diameters in which the fluid moves horizontally. The reactor achieved a uniform temperature profile by connecting three different furnaces in series to a heating zone $(45 \mathrm{~cm})$. Each reactor was controlled 
by six SiC heating elements monitored by three R-type thermocouples in direct contact with the outer surface of the tubular reactor. The reactivity of methane in the space between the furnaces was negligible. For safe operation, the system was installed in a container where negative pressure was maintained and could automatically shut off in case of gas leaks and pressure changes. The reactor reached the reaction temperature at a flow rate of $100 \mathrm{~mL} \mathrm{~min}^{-1}$ using He at a ramping rate of $10^{\circ} \mathrm{C} \mathrm{min}^{-1}$. Methane and methane/hydrogen gas containing $\mathrm{Ar}$ as an internal standard were then fed into the reactor using mass flow controllers (5850E, Brooks $\left.{ }^{\circledR}\right)$. All gases were passed through an oxygen/moisture trap (OT3-4, Agilent) before they were fed into the system to remove $\mathrm{O}_{2}$ and $\mathrm{H}_{2} \mathrm{O}$. To impede the condensation of aromatics, all lines from the reactor to the online gas chromatograph (GC) were heated to $190{ }^{\circ} \mathrm{C}$, and additional $\mathrm{H}_{2}$ was supplied to dilute the gas effluent from the reactor. We installed a $500 \mathrm{~mL}$ gas-solid separator and particle filters behind the reactor to prevent it from clogging due to coke. The gas analysis was carried out using an online GC (7820A, Agilent) equipped with two blocks of detectors composed of a thermal conductivity detector (TCD) and flame ionization detectors (FIDs). The molar concentrations of $\mathrm{H}_{2}, \mathrm{CH}_{4}, \mathrm{Ar}$, $\mathrm{CO}, \mathrm{CO}_{2}$, and $\mathrm{C} 2$ (ethane, ethylene, and acetylene) components were determined by the TCD with a ShinCarbon ST column (Restek Corp., Catalog No. 80486-800). The injection port and detector were maintained at $250^{\circ} \mathrm{C}$ and $200^{\circ} \mathrm{C}$, respectively. The column flow rate of the He carrier gas was $30 \mathrm{~mL} \mathrm{~min}^{-1}$. C3-C4 hydrocarbons and aromatics including benzene, naphthalene, and alkyl aromatics (toluene, xylenes, etc.) were separated using an Rtx-VMS column (Restek Corp., Catalog No. 49915). Their concentrations were measured using the FID. The injection port and detector were maintained at $200^{\circ} \mathrm{C}$ and $250^{\circ} \mathrm{C}$, respectively. The column flow rate of the He carrier gas was $0.5 \mathrm{~mL} \mathrm{~min}{ }^{-1}$. The GC oven temperature regime started off with a constant temperature 
of $50^{\circ} \mathrm{C}$ for $6 \mathrm{~min}$, followed by an increase to $200^{\circ} \mathrm{C}$ at $15^{\circ} \mathrm{C} \mathrm{min}^{-1}$, and finally maintained a constant temperature of $200^{\circ} \mathrm{C}$ for $8 \mathrm{~min}$.

The methane conversion was calculated as mole ratio of reacted methane to methane fed into the system. The product selectivity was calculated by the carbon amount of the product (mol) divided by the carbon amount of the reacted methane (mol). We calculate the coke selectivity according to the carbon balance at which the sum of the product selectivities detected by GC was subtracted from $100 \%$. The product yield was calculated by dividing the carbon amount (mol) of the product by the amount of fed methane (mol). These experimental results were tabulated in ESI (see Table S1).

\section{Target property prediction with ML}

To apply the ML approach, we reorganized the experimental data with six input variables and four target values. The input variables included (1) pressure $(p),(2)$ temperature $(T),(3)$ flow rate $(f)$, (4) $\mathrm{H}_{2}$ content $\left(H_{2}\right)$, and (5) length $\left(l_{R}\right)$ and (6) diameter $\left(d_{R}\right)$ of the reactors. The four target values were the (1) $\mathrm{C}_{2}$ yield, (2) $\mathrm{C}_{2}$ selectivity, (3) $\mathrm{CH}_{4}$ conversion, and (4) coke selectivity. After preparing the experimental data for the training of the ML models, we generated two ML models based on different algorithms such as fully-connected neural network (FNN) ${ }^{46,48}$ and XGBoost (abbreviated as XGB in this paper). ${ }^{47}$ In fact, FNN and XGB are used in various the chemistryrelated areas. ${ }^{49}$ FNN was employed as implemented in Tensorflow, ${ }^{50}$ while XGB was used as present in the XGBoost python package. By using the five-fold cross-validation, we searched the optimal hyperparameters of FNN by varying three factors: activation function, number of nodes, and optimizer. These parameters are listed in Table S2 in ESI. Scanning of possible combinations of hyperparameters showed that the combination of Sigmoid function, 60 nodes for two hidden 
layers, and Adadelta optimizer ${ }^{51}$ provided the best performance in the training of the FNN model for the prediction of the $\mathrm{C}_{2}$ selectivity. We targeted the $\mathrm{C}_{2}$ selectivity in hyperparameter optimization as it showed the largest error as shown later. We also performed the hyperparameter optimization for XGB models and selected 300 estimators and a maximum depth of 5. The range of the number of estimators and depth considered in this work are also listed in Table S2. For all cases, $80 \%$ of the experimental data were used as the training set to optimize model parameters, while the rest were treated as the test set for evaluations. Here, we randomly selected data for training and test sets and listed indices of test set (see Table S3 in ESI) as splitting training set and test set can influence the performance of the ML model.

\section{Global optimization with metaheuristics}

Metaheuristics is a widely used mathematical optimization method to find the optimal solution of a complex black-box model such as deep road networks, ${ }^{52}$ neural networks, ${ }^{53}$ and materials discovery, ${ }^{54,}{ }^{55}$ In this work, we adopted the artificial bee colony $(\mathrm{ABC})^{53}$ algorithm as a representative example of metaheustrics, which had been verified in several benchmarks ${ }^{56}$ and applied to various fields ${ }^{57}$ Figure 1 depicts the overall optimization process of the ABC algorithm, consisting mainly of four steps as follows: (1) Initial feature vectors (or population) are randomly generated based on a random seed, (2) each feasible solution is evaluated by the trained ML model and penalty function, (3) feasible solutions for the next trial are generated according to the algorithm-specific rules and solutions scored at the current stage, and finally (4) if the user-defined termination condition is satisfied, the optimization is terminated and the solution with the best score is returned. We repeated these optimization cycles 1000 times. The size of population was set to 100 . Notably, the optimization reached the converged solution after $\sim 100$ optimization cycles. 


\section{Results and Discussion}

The MTOAH was performed in a tubular quartz reactor with a crystalline surface as shown in Fig. 2 and Table S1. The methane conversion in the tubular reactor occurred through four main reaction steps: ${ }^{58}$ (1) initial activation of the $\mathrm{C}-\mathrm{H}$ bond in methane, (2) conversion of methyl radicals to ethane and subsequent dehydrogenations, (3) $\mathrm{C}-\mathrm{C}$ coupling of $\mathrm{C}_{2}$ species to aromatics, and (4) subsequent reactions for coke formation. Each reaction step is largely dependent on the concentration of unstable radicals, which can only remain for a period of microseconds in the reaction system. ${ }^{58}$ The $\mathrm{SiO}_{2}$ surface of the tubular reactor not only activates the $\mathrm{C}-\mathrm{H}$ bond of methane, but also acts as a coke terminator for unsaturated hydrocarbons. ${ }^{29}$ We adjusted several reaction parameters to change the free energies of the reaction, such as the temperature $\left(890-1200^{\circ} \mathrm{C}\right)$, pressure $(0.005-0.888 \mathrm{barg})$ and the reactant flow rate $\left(40-320 \mathrm{~mL} \mathrm{~min}^{-1}\right)$, reactor diameter $(4-16 \mathrm{~mm})$, and reactor length $(15-45 \mathrm{~cm})$. To improve the stability of unsaturated hydrocarbons during the entire reaction, we varied the $\mathrm{H}_{2}$ content in the feed, which was calculated as the mole ratio of $\mathrm{H}_{2}$ to $\mathrm{CH}_{4}$ and Ar. The yield of hydrocarbons was not thermodynamically limited. Only the $\mathrm{C}_{2}$ distribution of ethane, ethylene, and acetylene was near the equilibrium. Notably, the $\mathrm{C}_{2}$ selectivity altered by the reaction parameter tended to be inversely proportional to the coke selectivity. According to our experimental data used in this study, the quartz reactor achieved a $\mathrm{C}_{2}$ yield of $15.7 \%$; however, coke selectivity of $27.7 \%$ was observed as well (Table $\mathrm{S} 1$, Index 245). We found that by keeping the $\mathrm{C}_{2}$ yield in a parametric way, we systematically reduced the coke selectivity to $10.4 \%$ (Table S1, Index 249). To further improve the $\mathrm{C}_{2}$ yield, individual parameters must define their role. Therefore, ML is suitable for logically designing optimal operation conditions. 
Before the application of AI methods, we briefly analyzed the experimental data. As shown in Fig. 2, we visualized four important experimental quantities: $\mathrm{C}_{2}$ yield, $\mathrm{C}_{2}$ selectivity, $\mathrm{CH}_{4}$ conversion, and coke selectivity. As we focused on enhancing the $\mathrm{C}_{2}$ yield, a total of 250 experimental data were collected, and categorized into five groups according to the $\mathrm{C}_{2}$ yield in ascending order. We assigned indices to these data. Thus, index 250 is the experimental result with the highest $C_{2}$ yield, while index 1 corresponds to the lowest $C_{2}$ yield. Fig. 2 shows that the $\mathrm{C}_{2}$ yield varied in the range of $\sim 0$ to $\sim 15 \%$. Complex relationships were observed between other quantities and these indices. We analyzed the $\mathrm{C}_{2}$ yield, according to the input variables such as (1) pressure, (2) temperature, (3) flow rate, (4) $\mathrm{H}_{2}$ content, and (5) length and (6) diameter of the reactor, as shown in Fig. 3. Out of these, only temperature was weakly related to the $\mathrm{C}_{2}$ yield, which was almost independent of the other experimental input variables. Therefore, we can expect that ML methods can be used to determine the complex relationships between the experimental input and output variables.

In Fig. 4, the ML models based on FNN and XGB predicted the experimental results within a mean absolute error (MAE) of 5\%. Overall, XGB provides better prediction results than FNN. As it is challenging to generate high-quality experimental data for a specific purpose, the applicability of XGB to the small dataset generated from our experimental results is valuable. With these, we adopted XGB for the further analysis in this work and displayed prediction results for four target quantities as shown in Fig. 5. We can explain relatively large MAEs for $\mathrm{C}_{2}$ and coke selectivities based on several outliers in the prediction of these quantities, depicted in Fig. 5(b) and 5(d). In addition, XGB can rank input features based on their relative importance in the construction of the ML as shown in Fig. 6. This is a valuable characteristic of XGB because it records the contribution of each feature during the training step, unlike the typical FNN. The 
reaction temperature is the most important variable in the prediction of $\mathrm{C}_{2}$ yield as the temperature was roughly related to the $\mathrm{C}_{2}$ yield as shown in Fig. 3(b). Other variables also participated to improve the XGB model. Their relative importance values ranged between $5-20 \%$. The relative contributions of the temperature and pressure are different for MTOAH and OCM as the pressure exhibits the highest importance among several experimental parameters of OCM. ${ }^{17}$ For the $\mathrm{C}_{2}$ and coke selectivities, pressure was chosen as the most important factor among the input variables although the $\mathrm{C}_{2}$ selectivity is also considerably affected by the flow rate and $\mathrm{H}_{2}$ content. According to relative importances from the XGB algorithm, the prediction of methane conversion was mostly influenced by the flow rate. By comparing the contributions of each input variable, we can propose a simple guide to optimize the reaction conditions for MTOAH as the modulation of temperature and pressure is important to increase the $\mathrm{C}_{2}$ yield and $\mathrm{C}_{2}$ selectivity, respectively.

We employed the metaheuristics-based optimization to evaluate the experimental conditions for maximum $\mathrm{C}_{2}$ yield. As explained above, the optimization algorithm utilizes the trained XGB model to predict MTOAH results based on our experimental data. During the optimization, we restricted the range of experimental input parameters such as temperature $\left(850-1250^{\circ} \mathrm{C}\right)$, pressure $(0-1 \mathrm{barg})$, the reactant flow rate $\left(10-350 \mathrm{~mL} \mathrm{~min}^{-1}\right)$, the $\mathrm{H}_{2}$ content $(0-$ 5.5), reactor diameter $(3-18 \mathrm{~mm})$, and reactor length $(10-50 \mathrm{~cm})$. These ranges of parameters are experimentally feasible as they are $\sim 10 \%$ beyond the parameters that were used in the data generation. Notably, the algorithm proposes a feasible solution based on AI without relying on additional experiments. Figure 7 shows XGB-model-based predicted values for the $\mathrm{C}_{2}$ yield and coke selectivity under the optimized experimental conditions. The x-axis represents the number of data. We show the maximum and average values of the experimental data for each case. For the coke selectivity, it is meaningless to show the maximum because the value was larger than $80 \%$ 
even with the first 50 experimental data. As we reorganized experimental data based on the $\mathrm{C}_{2}$ yield in ascending order, the $\mathrm{C}_{2}$ yield increased with the number of data. Therefore, training the XGB model with more and more data means that the data with higher $\mathrm{C}_{2}$ yield is used for training. The results obtained with the XGB models with different numbers of experimental data were varied. For all tested cases, our AI-based optimization improved the $\mathrm{C}_{2}$ yield from $8.03 \%$ to $18.10 \%$ when compared with the maximum $\mathrm{C}_{2}$ yield obtained by experiments. However, the formation of coke, a by-product of MTOAH, also increased with the increase in the $\mathrm{C}_{2}$ yield.

The ideal optimization should maximize the yield of $\mathrm{C}_{2}$ compounds and their selectivity, and thus minimize the selectivity for the coke. The optimization of multiple target quantities is an important issue in catalytic reaction engineering. ${ }^{59,60} \mathrm{We}$ devised a set of scoring functions by taking the geometric mean of linear functions of three quantities, which are $\mathrm{CH}_{4}$ conversion, $\mathrm{C}_{2}$ selectivity, and coke selectivity. These linear functions have been shown in Fig. 8. It should be noted that there coule be enumorous possibilities to construct scoring functions and we selected one of them. Here, we use the $\mathrm{C}_{2}$ selectivity, as a high $\mathrm{C}_{2}$ selectivity is roughly related to a low coke selectivity. The yield of $\mathrm{C}_{2}$ compounds and their selectivities were scored linearly from 0 to 1. For the coke selectivity, we approached in the opposite way and introduced a cutoff. If the coke selectivity becomes higher than the cutoff, the score is set to 0 . We tested four cutoffs for the coke selectivity as shown in Fig. 8(b). Using these, we could change the optimization of three target quantities to that of a single quantity called the scoring function. Our aim is to maximize the value of these functions instead of the $\mathrm{C}_{2}$ yield because they enable us to consider the $\mathrm{C}_{2}$ yield and coke selectivity simultaneously. Figure 9 shows the $\mathrm{C}_{2}$ yield and coke selectivity obtained by optimizing the scoring functions. These results support the finding that a reduction in the cutoff increases the penalty for the coke formation. Therefore, the AI can find the experimental conditions to optimize 
the $\mathrm{C}_{2}$ yield with a smaller amount of coke formation. When the cutoff for the coke was $40 \%$, the XGB model predicted a $\mathrm{C}_{2}$ yield of $13.97 \%$ and coke selectivity of $3.76 \%$. As the MAE of the coke selectivity was $2.78 \%$, the coke selectivity already reached the prediction limit in this case and we could not reduce the cutoff below $40 \%$. This experimental condition was further used to validate our AI approach with an additional MTOAH experiment, as shown in Table 1. Although some mismatches in experimental variables existed in practice, we could achieve a $\mathrm{C}_{2}$ yield of $10.52 \%$ with a low coke selectivity of $1.10 \%$. Five cases (Indices of 172, 175, 184, 186, and 188) in Table $\mathrm{S} 1$ show similar $\mathrm{C}_{2}$ yields to this results, but the coke selectivities were $3.2-4.8 \%$ in these cases, which were higher than the experimental values in Table 1 . We also tabulated these experimental data in Table S4 in ESI. In our experiment, 73 practices provided $\mathrm{C}_{2}$ yields above $10.52 \%$; however, they mostly exhibited coke selectivity above $6 \%$. Considering the prediction errors of ML models ( $\sim 3 \%$ in this study), we confirm that AI suggestions can predict experimental conditions with better results. As quantifying the uncertainty from ML prediction is an active research topic, ${ }^{61,62}$ quantitative error analysis could be helpful for improving AI suggestions in the future. From the experimental perspective, with this Al's reasonable prediction, we expect that complex reaction mechanisms of MTOAH will be further optimized with cascade reactors containing different parameters for better $\mathrm{C}_{2}$ yield as well as $\mathrm{C}_{2} \mathrm{H}_{4}$ yield while maintaining negligible coke selectivity. Furthermore, it is also necessary to develop AI approaches and accumulate refined data for more accurate ML models, which can enhance AI suggestions to improve catalytic processes such as MTOAH for application in the chemical industry. 
Table 1. Experimental conditions and outcomes of the AI suggestion and additional experiment.

\begin{tabular}{lcccccccc}
\hline & $p($ barg $)$ & $T\left({ }^{\circ} \mathrm{C}\right)$ & $f(\mathrm{sccm})$ & $\begin{array}{c}\mathrm{H}_{2} \\
\text { content }\end{array}$ & $l_{R}(\mathrm{~cm})$ & $\begin{array}{c}d_{R} \\
(\mathrm{~mm})\end{array}$ & $\begin{array}{c}\mathrm{C}_{2} \text { yield } \\
(\%)\end{array}$ & $\begin{array}{c}\text { Coke } \\
\text { selectivity } \\
(\%)\end{array}$ \\
\hline AI & 0.16 & 1196.3 & 148.7 & 3.6 & 10.6 & 6.4 & 13.97 & 3.76 \\
Exp. & 0.13 & 1196.3 & 148.8 & 3.6 & 15.0 & 6.5 & 10.52 & 1.10 \\
\hline
\end{tabular}

\section{Conclusions}

In summary, we propose an $\mathrm{AI}$ approach to optimize the MTOAH reaction to $\mathrm{C}_{2}$ compounds using ML for regression and metaheuristics for global optimization. We experimentally generated MTOAH data and then applied two well-known ML methods, XGB and FNN. As these ML models can predict the experimental results with errors of $<5 \%$ and XGB works better than FNN, we further employed the trained XGB models. In addition, we can take advantage of using XGB models as they can provide a qualitative guideline for better experimental results based on the relative importance of the input variables. We can also propose the experimental conditions for enhancing the $\mathrm{C}_{2}$ yield in a quantitiative manner by adopting $\mathrm{ABC}$ algorithm, which is a metaheuristic method. However, we observed that focusing on the $\mathrm{C}_{2}$ yield only will increase the production of the coke, a side product in this work. To optimize both $\mathrm{C}_{2}$ yield and the coke selectivity at the same time, we devised scoring functions and found experimental conditions suggested by $\mathrm{ABC}$ algorithm. We experimentally verified that the suggested condition can indeed provide a reasonable $\mathrm{C}_{2}$ yield with a low coke selectivity. With these, we believe that our approach can be applied to optimize various chemical reactions in the future. 


\section{Acknowledgments}

This research was financially supported by C1 Gas Refinery Program through the National Research Foundation of Korea (NRF) funded by the Ministry of Science, ICT and Future Planning (NRF-2017M3D3A1A01037001). This study was also supported by a core Korea Research Institute of Chemical Technology (KRICT) project (SI2051-10). 


\section{References}

1. J. Hagen, Industrial catalysis: a practical approach, John Wiley \& Sons, 2015.

2. R. J. Hendershot, C. M. Snively and J. Lauterbach, Chem. Eur. J., 2005, 11, 806-814.

3. D. Farrusseng, Surf. Sci. Rep., 2008, 63, 487-513.

4. U. Zavyalova, M. Holena, R. Schlögl and M. Baerns, ChemCatChem, 2011, 3, 19351947.

5. E. Kim, K. Huang, A. Saunders, A. McCallum, G. Ceder and E. Olivetti, Chem. Mater., 2017, 29, 9436-9444.

6. G. Rothenberg, Catal. Today, 2008, 137, 2-10.

7. T. C. Le and D. A. Winkler, Chem. Rev., 2016, 116, 6107-6132.

8. J. E. Kreutz, A. Shukhaev, W. Du, S. Druskin, O. Daugulis and R. F. Ismagilov, J. Am. Chem. Soc., 2010, 132, 3128-3132.

9. S. Moehmel, N. Steinfeldt, S. Engelschalt, M. Holena, S. Kolf, M. Baerns, U.

Dingerdissen, D. Wolf, R. Weber and M. Bewersdorf, Appl. Catal. A, 2008, 334, 73-83.

10. K. Suzuki, T. Toyao, Z. Maeno, S. Takakusagi, K. i. Shimizu and I. Takigawa, ChemCatChem, 2019, 11, 4537-4547.

11. A. D. Clayton, A. M. Schweidtmann, G. Clemens, J. A. Manson, C. J. Taylor, C. G. Niño, T. W. Chamberlain, N. Kapur, A. J. Blacker, A. A. Lapkin and R. A. Bourne, Chem. Eng. J., 2020, 384, 123340.

12. H. Gao, T. J. Struble, C. W. Coley, Y. Wang, W. H. Green and K. F. Jensen, ACS Cent. Sci., 2018, 4, 1465-1476.

13. M. E. Günay and R. Yildirim, ChemCatChem, 2013, 5, 1395-1406.

14. Ç. Odabaşı, M. E. Günay and R. Yıldırım, Int. J. Hydrog. Energy, 2014, 39, 5733-5746. 
15. M. Baysal, M. E. Günay and R. Yıldırım, Int. J. Hydrog. Energy, 2017, 42, 243-254.

16. J. Ohyama, S. Nishimura and K. Takahashi, ChemCatChem, 2019, 11, 4307-4313.

17. K. Takahashi, I. Miyazato, S. Nishimura and J. Ohyama, ChemCatChem, 2018, 10, 32233228.

18. L. Pirro, P. S. Mendes, B. D. Vandegehuchte, G. B. Marin and J. W. Thybaut, React. Chem. Eng., 2020, 5, 584-596.

19. R. Schmack, A. Friedrich, E. V. Kondratenko, J. Polte, A. Werwatz and R. Kraehnert, Nat. Commun., 2019, 10, 441

20. T. N. Nguyen, T. T. P. Nhat, K. Takimoto, A. Thakur, S. Nishimura, J. Ohyama, I. Miyazato, L. Takahashi, J. Fujima and K. Takahashi, ACS Catal., 2019, 10, 921-932.

21. I. Miyazato, S. Nishimura, L. Takahashi, J. Ohyama and K. Takahashi, J. Phys. Chem. Lett., 2020, 11, 787-795.

22. S. Faramawy, T. Zaki and A.-E. Sakr, J. Nat. Gas Sci. Eng., 2016, 34, 34-54.

23. M. Montingelli, S. Tedesco and A. Olabi, Renew. Sust. Energy Rev., 2015, 43, 961-972.

24. R. Chandra, H. Takeuchi and T. Hasegawa, Renew. Sust. Energy Rev., 2012, 16, 14621476.

25. A. Iulianelli, S. Liguori, J. Wilcox and A. Basile, Catal. Rev., 2016, 58, 1-35.

26. N. A. K. Aramouni, J. G. Touma, B. A. Tarboush, J. Zeaiter and M. N. Ahmad, Renew. Sust. Energy Rev., 2018, 82, 2570-2585.

27. J. H. Lunsford, Angew. Chem. Int. Ed., 1995, 34, 970-980.

28. Y. Xu, X. Bao and L. W. Lin, J. Catal., 2003, 216, 386-395.

29. S. J. Han, S. W. Lee, H. W. Kim, S. K. Kim and Y. T. Kim, ACS Catal., 2019, 9, 79847997. 
30. X. Guo, G. Fang, G. Li, H. Ma, H. Fan, L. Yu, C. Ma, X. Wu, D. Deng and M. Wei, Science, 2014, 344, 616-619.

31. P. Tang, Q. Zhu, Z. Wu and D. Ma, Energy Environ. Sci., 2014, 7, 2580-2591.

32. H. Amariglio, J. Saint-Just and A. Amariglio, Fuel Process. Technol., 1995, 42, 291-323.

33. L. Guczi, K. Sarma and L. Borkó, Catal. Lett., 1996, 39, 43-47.

34. H. Sheng, E. P. Schreiner, W. Zheng and R. F. Lobo, ChemPhysChem, 2018, 19, 504511.

35. F. Amano, C. Akamoto, M. Ishimaru, S. Inagaki and H. Yoshida, Chem. Commun., 2020, 56, 6348-6351.

36. X. Yu, V. L. Zholobenko, S. Moldovan, D. Hu, D. Wu, V. V. Ordomsky and A. Y. Khodakov, Nat. Energy, 2020, 1-9.

37. J. Kim, J. Jeoung, J. Jeon, J. Kim, Y. S. Mok and K.-S. Ha, Chem. Eng. J., 2019, 377, 119896.

38. M. Scapinello, E. Delikonstantis and G. D. Stefanidis, Chem. Eng. Process., 2017, 117, $120-140$.

39. A. Holmen, Catal. Today, 2009, 142, 2-8.

40. P. Xie, T. Pu, A. Nie, S. Hwang, S. C. Purdy, W. Yu, D. Su, J. T. Miller and C. Wang, ACS Catal., 2018, 8, 4044-4048.

41. Y. Xiao and A. Varma, ACS Catal., 2018, 8, 2735-2740.

42. S. C. Oh, E. Schulman, J. Zhang, J. Fan, Y. Pan, J. Meng and D. Liu, Angew. Chem., 2019, 131, 7157-7160.

43. M. Sakbodin, Y. Wu, S. C. Oh, E. D. Wachsman and D. Liu, Angew. Chem., 2016, 128, $16383-16386$. 
44. S. K. Kim, H. W. Kim, S. J. Han, S. W. Lee, J. Shin and Y. T. Kim, Commun. Chem,, $2020,3,58$.

45. Z. Beheshti and S. M. H. Shamsuddin, Int. J. Adv. Soft Comput. Appl, 2013, 5, 1-35.

46. I. Goodfellow, Y. Bengio and A. Courville, Deep learning, MIT press, 2016.

47. T. Chen and C. Guestrin, 2016.

48. Y. LeCun, Y. Bengio and G. Hinton, Nature, 2015, 521, 436-444.

49. Z. Wu, B. Ramsundar, E. N. Feinberg, J. Gomes, C. Geniesse, A. S. Pappu, K. Leswing and V. Pande, Chem. Sci., 2018, 9, 513-530.

50. M. Abadi, P. Barham, J. Chen, Z. Chen, A. Davis, J. Dean, M. Devin, S. Ghemawat, G. Irving and M. Isard, 2016.

51. M. D. Zeiler, arXiv preprint arXiv:1212.5701, 2012.

52. S. B. Jha, J. Jha and M. K. Tiwari, Comput. Ind. Eng., 2019, 130, 166-186.

53. D. Karaboga, B. Akay and C. Ozturk, 2007.

54. P. C. Jennings, S. Lysgaard, J. S. Hummelshøj, T. Vegge and T. Bligaard, npj Comput. Mater., 2019, 5, 46.

55. S. Wu, Y. Kondo, M.-a. Kakimoto, B. Yang, H. Yamada, I. Kuwajima, G. Lambard, K. Hongo, Y. Xu and J. Shiomi, npj Comput. Mater., 2019, 5, 66.

56. D. Karaboga and B. Akay, Appl. Math. Comput., 2009, 214, 108-132.

57. D. Karaboga, B. Gorkemli, C. Ozturk and N. Karaboga, Artif. Intell. Rev., 2014, 42, 21 57.

58. A. Holmen, O. Olsvik and O. Rokstad, Fuel Process. Technol., 1995, 42, 249-267.

59. C. Mateos, M. J. Nieves-Remacha and J. A. Rincón, React. Chem. Eng., 2019, 4, 15361544. 
60. A. D. Clayton, J. A. Manson, C. J. Taylor, T. W. Chamberlain, B. A. Taylor, G. Clemens and R. A. Bourne, React. Chem. Eng., 2019, 4, 1545-1554.

61. A. A. Peterson, R. Christensen and A. Khorshidi, Phys. Chem. Chem. Phys., 2017, 19, 10978-10985.

62. J. P. Janet, C. Duan, T. Yang, A. Nandy and H. J. Kulik, Chem. Sci., 2019, 10, 79137922. 


\section{Figures}

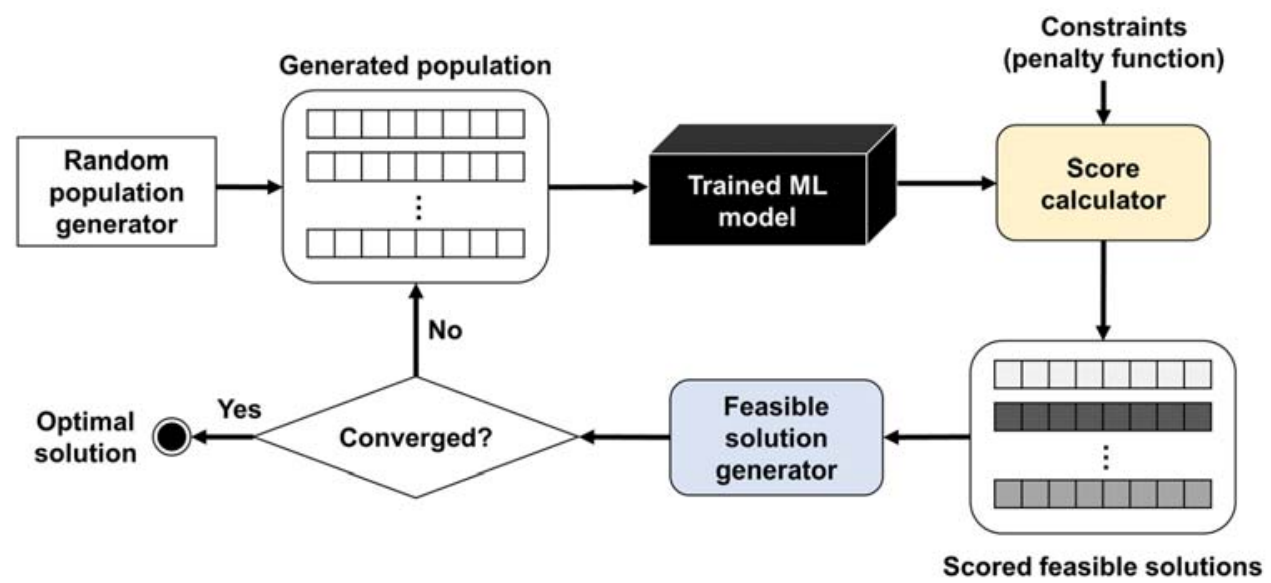

Figure 1. Schematic of the metaheuristic optimization. 

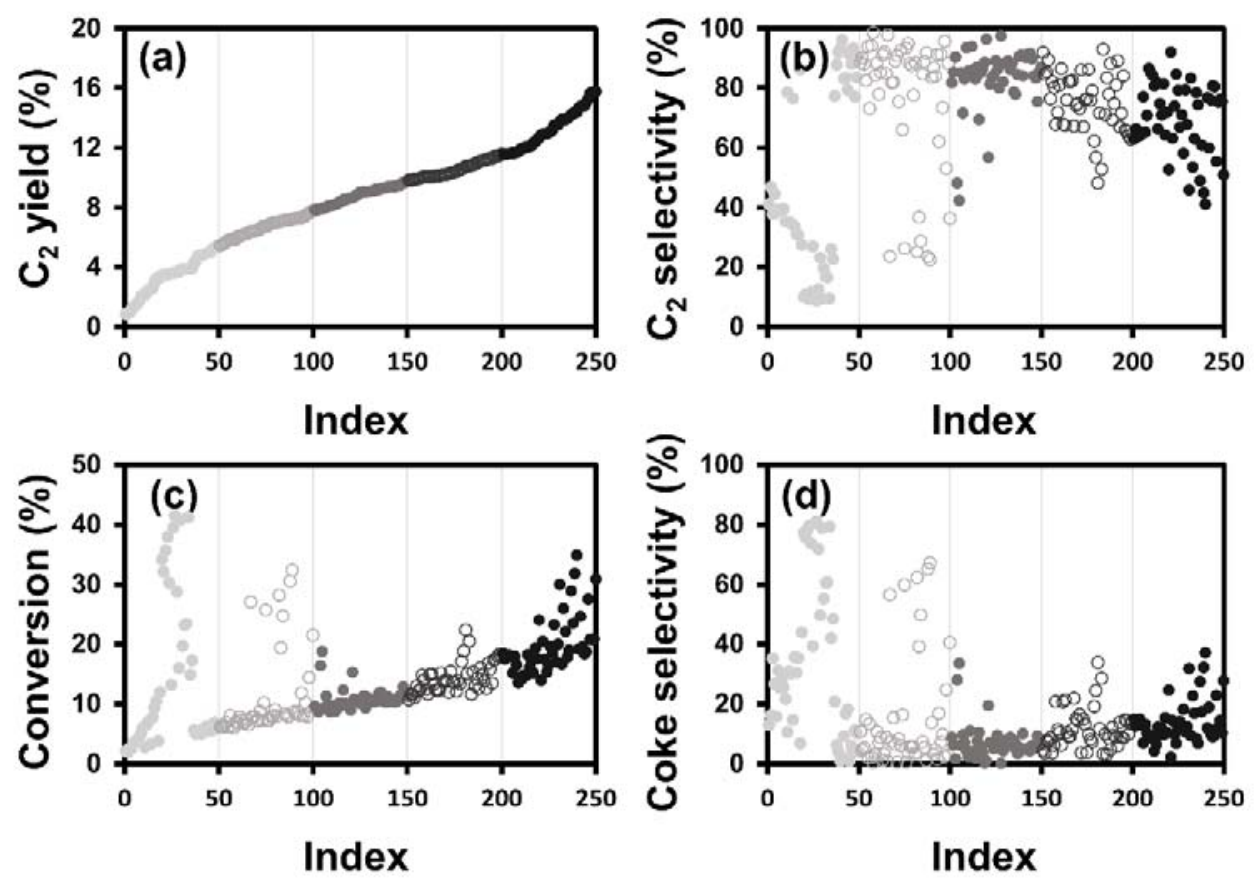

Figure 2. Distribution of experimental data for the (a) $\mathrm{C}_{2}$ yield, (b) $\mathrm{C}_{2}$ selectivity, (c) $\mathrm{CH}_{4}$ conversion, and, (d) coke selectivity. We classified the data for $\mathrm{C}_{2}$ yield into five groups in ascending order. Grouped data with higher yields are shown by darker colors. 

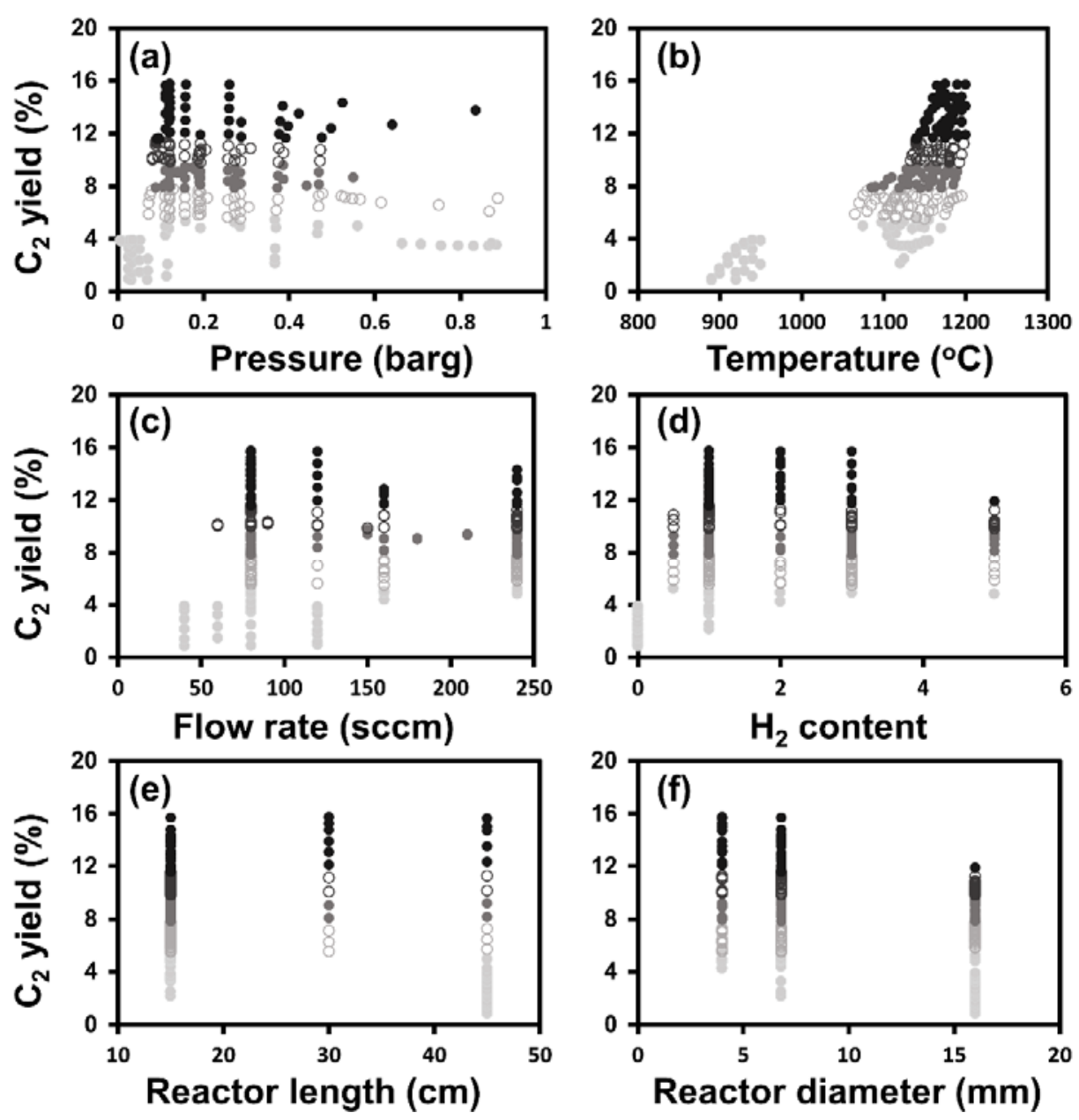

Figure 3. Experimental input parameters for $\mathrm{C}_{2}$ yield. The color scheme is the same as in Fig. 2 . 


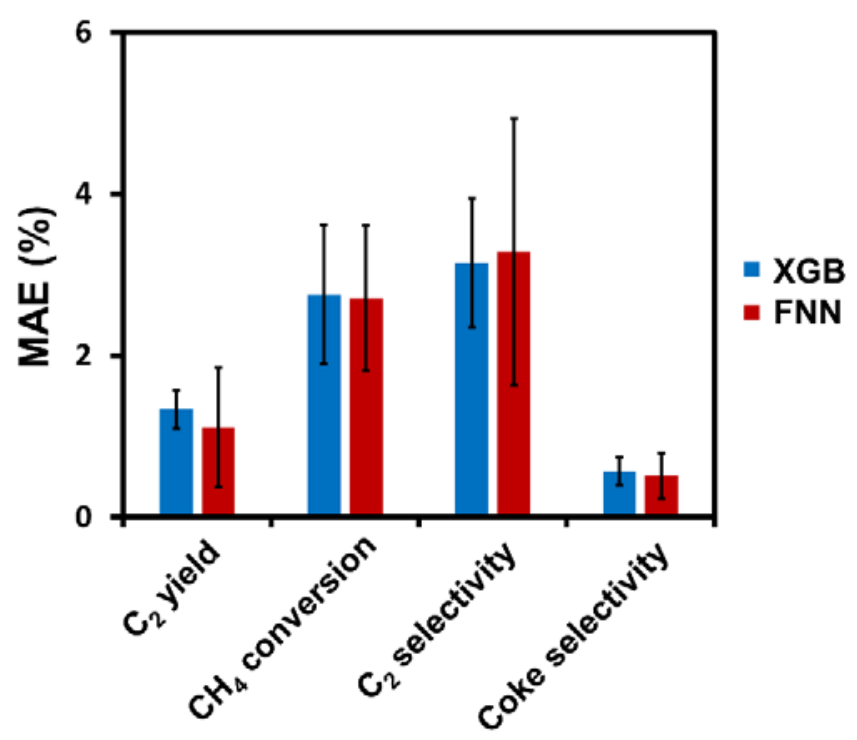

Figure 4. Prediction errors of the two ML models (XGB and FNN) for the experimental results: $\mathrm{C}_{2}$ yield, $\mathrm{CH}_{4}$ conversion, $\mathrm{C}_{2}$ selectivity, and coke selectivity. We measured the prediction error five times by changing the number of data by 50 , from 50 to 250 . The average of these errors and their standard deviation are displayed. 


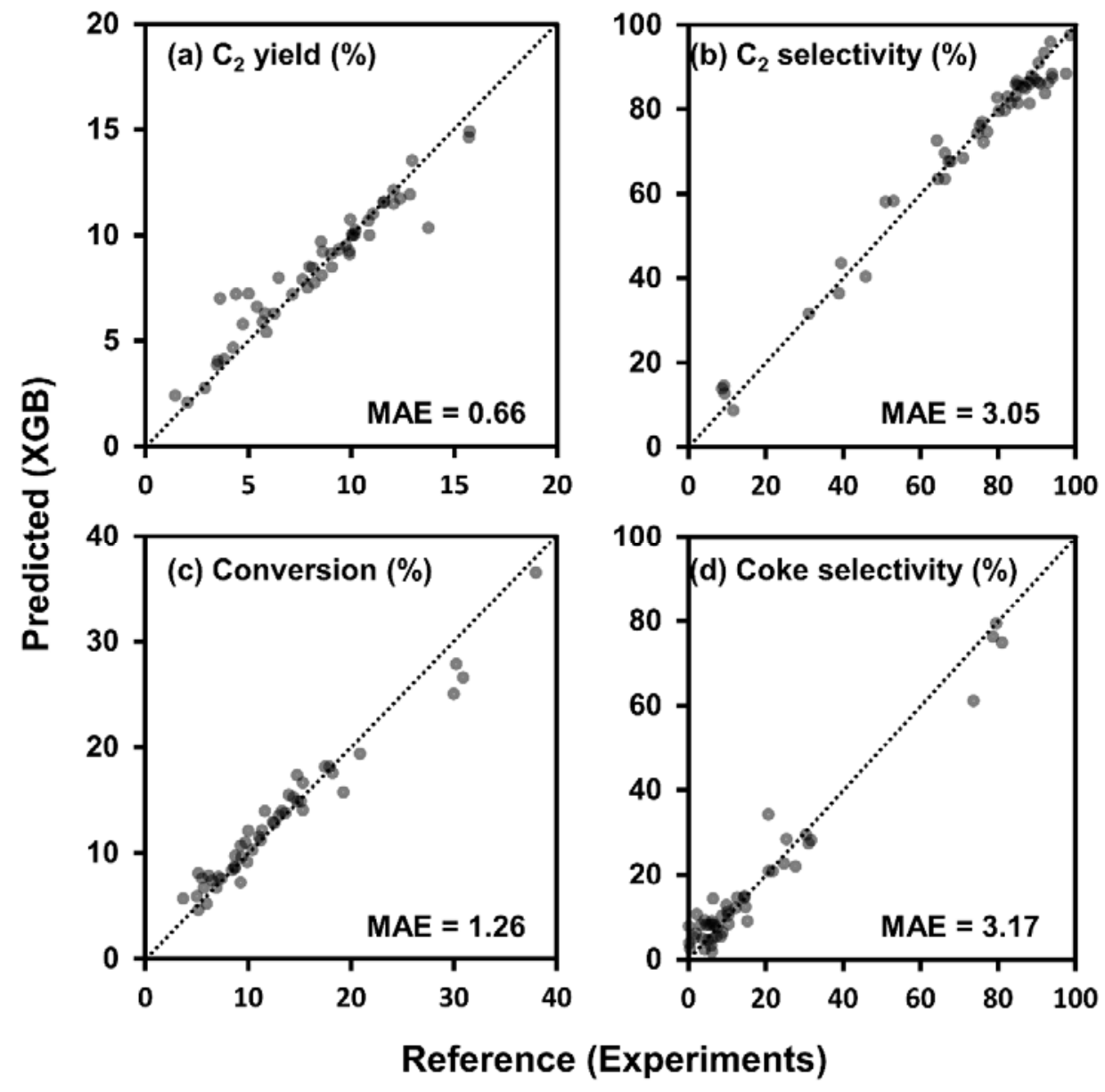

Figure 5. Comparison of the predicted and reference data with corresponding MAE for (a) $\mathrm{C}_{2}$ yield, (b) $\mathrm{C}_{2}$ selectivity, (c) $\mathrm{CH}_{4}$ conversion, and (d) coke selectivity. 

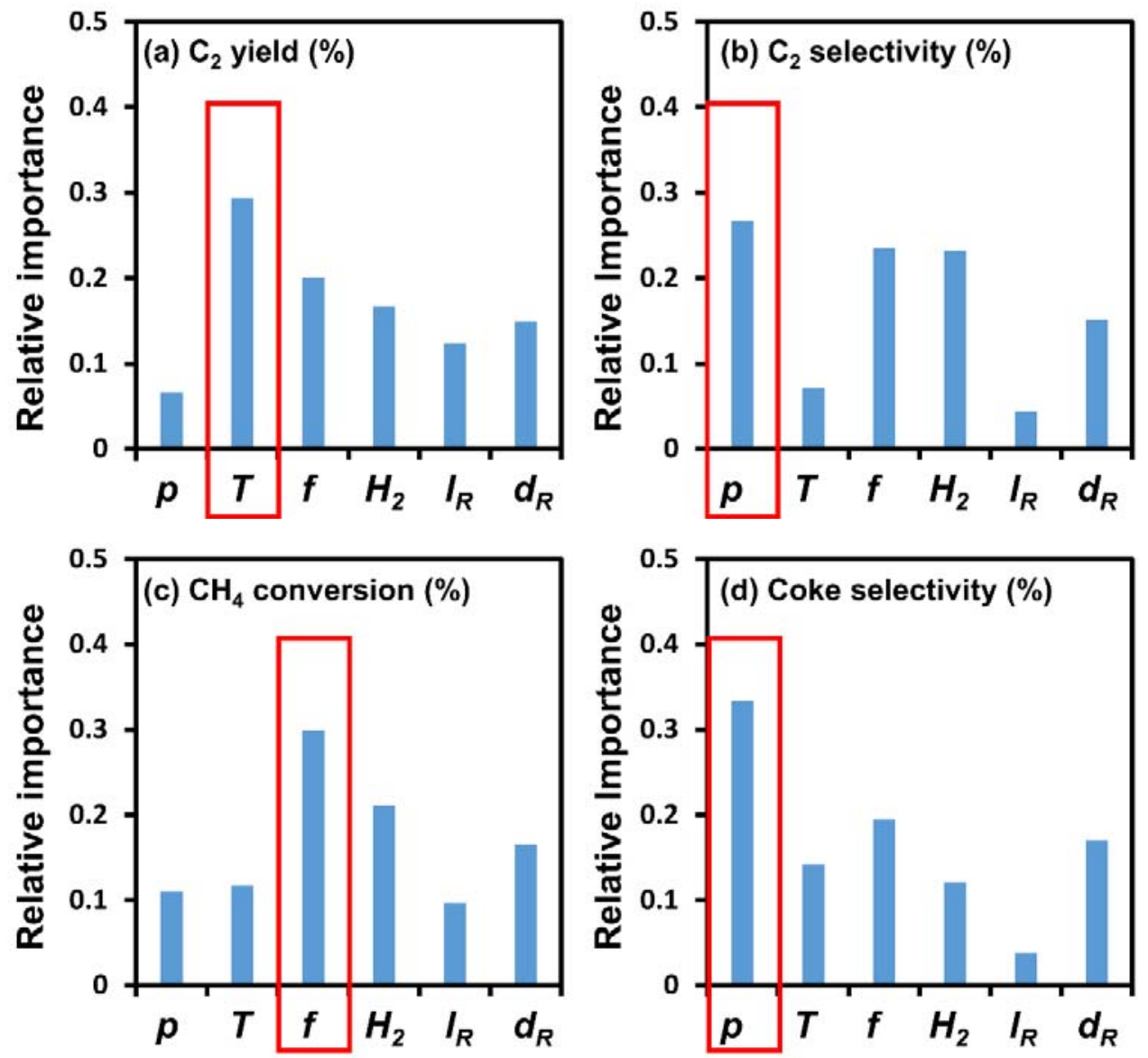

Figure 6. Relative importance values of the experimental input variables used for building ML models to predict the (a) $\mathrm{C}_{2}$ yield, (b) $\mathrm{C}_{2}$ selectivity, (c) $\mathrm{CH}_{4}$ conversion, and (d) coke selectivity. The most important variable is highlighted with a red box. 

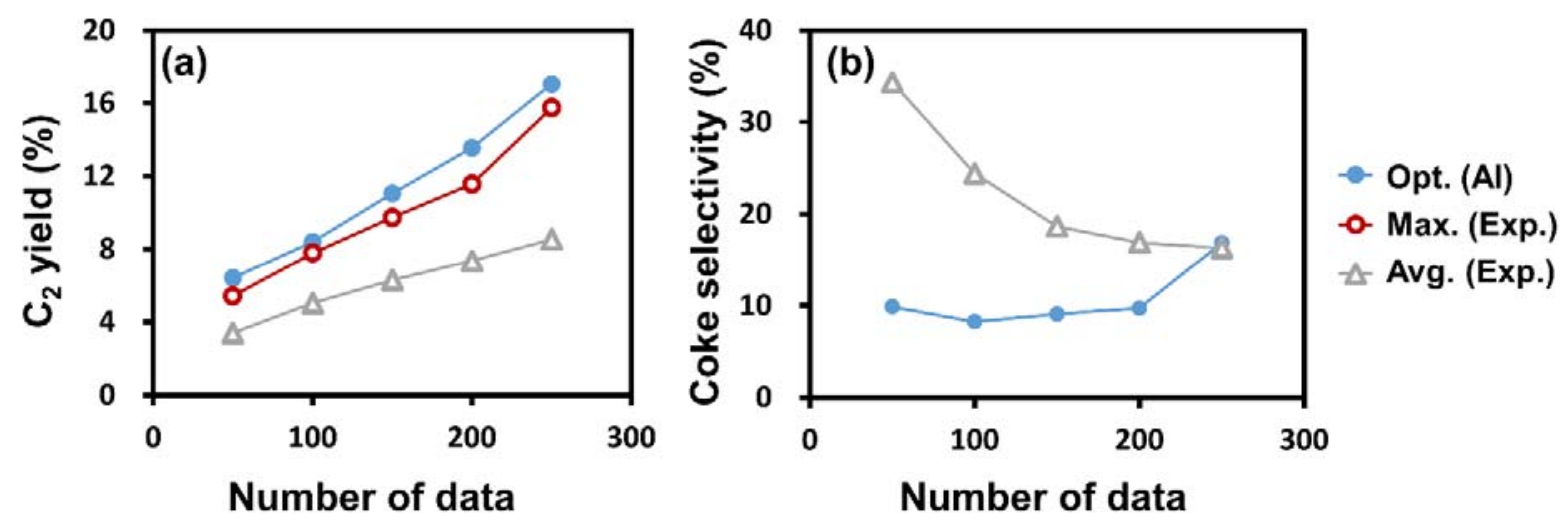

Figure 7. Results of the AI-based optimization and experiments for the (a) $\mathrm{C}_{2}$ yield and (b) coke selectivity. The AI optimization results (filled blue circles) are compared to the maximum value of the experimental results (open red circles) and their average (open gray triangles). 

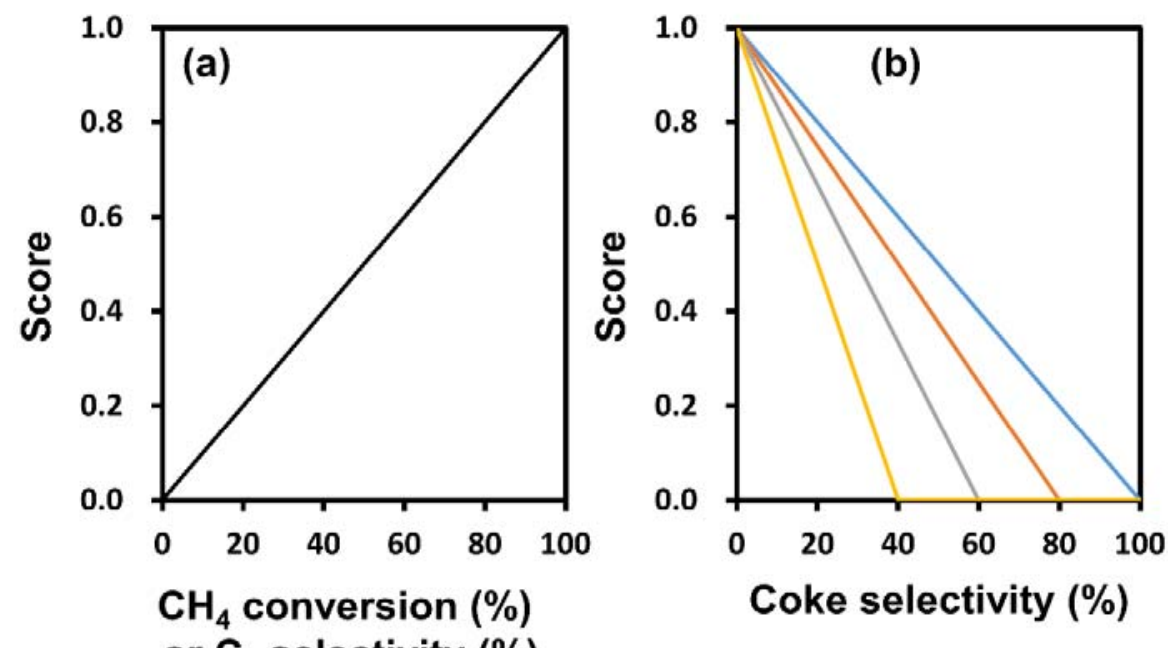

Figure 8. Scoring functions for the (a) $\mathrm{CH}_{4}$ convsersion and $\mathrm{C}_{2}$ selectivity and (b) coke selectivity. In (b), the cutoff values for the coke selectivity are set to $40 \%$ (yellow), $60 \%$ (gray), $80 \%$ (orange), and $100 \%$ (blue). 


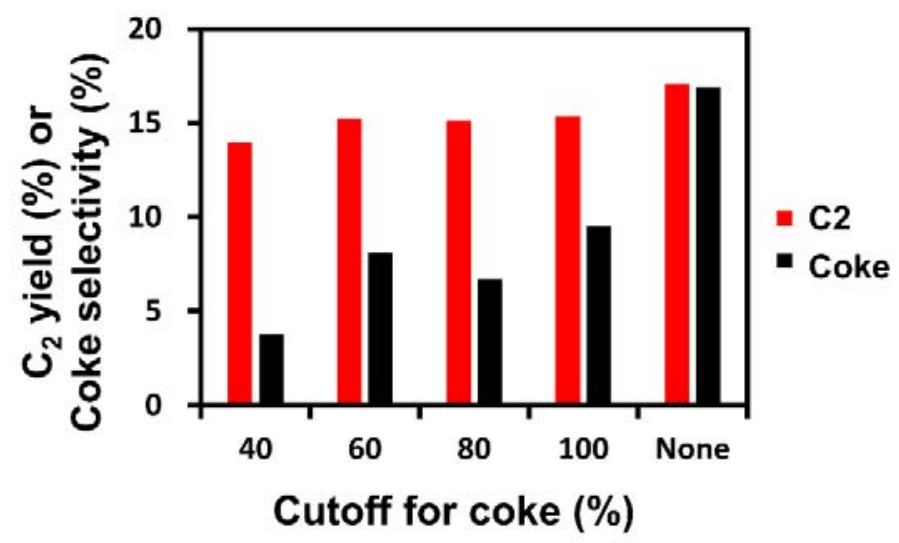

Figure 9. Effects of the cutoff for coke selectivity on the $\mathrm{C}_{2}$ yield (red) and coke selectivity (black). 